\title{
Evaluation of pharmacokinetic, pharmacodynamic, efficacy, and safety data of low-dose ticagrelor versus standard dose in East Asians: a systematic review
}

This article was published in the following Dove Press journal:

Therapeutics and Clinical Risk Management

\author{
Yun Jeong Lee' \\ Hyewon Kim' \\ Jiyeon Choi \\ Bo Hee Lee' \\ Seok-Yong Lee ${ }^{2}$ \\ 'Department of Pharmacy, College \\ of Pharmacy, Dankook University, \\ Cheonan, Republic of Korea; \\ ${ }^{2}$ Department of Pharmacy, School of \\ Pharmacy, Sungkyunkwan University, \\ Suwon, Republic of Korea
}

\begin{abstract}
East Asians are prone to higher systemic exposure and increased risk of bleeding compared to other races after administration of antiplatelet agent(s). The aim of this systematic review was to compare and evaluate the appropriateness of a lower dose versus standard dose of ticagrelor in East Asians. A systematic search was conducted in PubMed, EMBASE, and Cochrane databases for studies comparing low versus standard doses of ticagrelor in East Asian populations; a total of seven studies were included in the review. Pharmacokinetic (PK), pharmacodynamic (PD), clinical efficacy, and safety data were collected. PK data demonstrated that the $\mathrm{C}_{\max }$ and area under the curve (AUC) of ticagrelor increased dose-proportionally. PD data indicated that while the low dose of ticagrelor resulted in less antiplatelet activity in three of the five PD studies, in the other studies, there were no differences between low and standard doses. There were two clinical studies included in this review, which showed that the efficacy was similar between the low and standard doses, but both studies were limited by the number of patients included. While there were generally greater incidence of adverse events observed in the standard ticagrelor dose, the magnitude or actual difference between the two doses is difficult to determine due to lack of consistent data. In East Asians, the appropriateness of the low-dose ticagrelor cannot be determined based on the currently available evidence. Additional large-scale and longer duration studies are warranted.

Keywords: ticagrelor, P2Y12 receptor antagonist, lower dose, East Asians, Korean, Japanese, Chinese, efficacy, safety
\end{abstract}

\section{Introduction}

Ticagrelor is an oral, reversibly binding, direct-acting $\mathrm{P} 2 \mathrm{Y}_{12}$ receptor antagonist. It is approved worldwide for use in acute coronary syndrome (ACS) patients in combination with low-dose aspirin at a loading dose (LD) of $180 \mathrm{mg}$, followed by maintenance dose (MD) of $90 \mathrm{mg}$ twice daily. In addition, based on the PEGASUS-TIMI 54 study, ${ }^{1}$ ticagrelor has been approved at doses of $60 \mathrm{mg}$ twice daily in selected patients of post-myocardial infarction (MI) of one or more years.

Ticagrelor is rapidly absorbed after oral administration and has a predictable pharmacokinetic (PK) profile. ${ }^{2}$ Ticagrelor gets metabolized by the cytochrome P450 (CYP) enzyme to AR-C124910XX, a metabolite that possesses equivalent antiplatelet potency as the parent drug. ${ }^{3,4}$ Both ticagrelor and AR-C124910XX bind reversibly to the $\mathrm{P} 2 \mathrm{Y}_{12}$ receptor and exert their pharmacologic effects. ${ }^{5}$ The most common adverse events of ticagrelor are bleeding, dyspnea, gastrointestinal disturbances such as nausea and diarrhea, ventricular pauses $\geq 3 \mathrm{sec}$, and bradycardia. ${ }^{6,7}$ 
In the PLATO trial, ticagrelor has been compared to clopidogrel for prevention of cardiovascular (CV) events in ACS patients. Ticagrelor was more effective at reducing the composite rate of MI, stroke, or death from vascular causes at 12 months and it had similar rates of major bleeding compared to clopidogrel, but more non-coronary artery bypass grafting-related major bleeding and combined major or minor bleeding were observed. ${ }^{6}$

In PHILO, a study designed similar to PLATO but aimed to assess the outcomes of East Asian patients, 801 East Asian patients with ACS who were planned to undergo percutaneous coronary intervention (PCI) were followed up for a mean of 200-210 days. This study found that there were no significant difference between ticagrelor and clopidogrel in clinical efficacy and in major bleeding. However, minor bleeding and composite of major and minor bleeding occurred at a higher rate in the ticagrelor group. ${ }^{8}$ From this study, superior efficacy of ticagrelor, as observed in the PLATO trial, was questioned in East Asian patients, and more alarmingly, the risk of higher bleeding rates in the ticagrelor group suggested a need for further evaluation of a lower dose of ticagrelor in East Asian population. ${ }^{9}$

Of note, in Japan, other widely used oral $\mathrm{P} 2 \mathrm{Y}_{12}$ receptor antagonists such as clopidogrel and prasugrel have been approved at lower doses than the doses approved worldwide based on the PK, pharmacodynamic (PD), and clinical studies of these agents in the Japanese population. ${ }^{10-15}$ Clopidogrel, which is approved at MD of $75 \mathrm{mg}$ daily worldwide, is also approved at $75 \mathrm{mg}$ daily in Japan, but it is suggested that $50 \mathrm{mg}$ daily dose can also be used based on patient's age, body weight, and symptoms. ${ }^{16}$ Prasugrel, which is approved at LD of $60 \mathrm{mg}$ and MD of $10 \mathrm{mg}$ daily worldwide, is approved at $20 \mathrm{mg} \mathrm{LD}$ and $3.75 \mathrm{mg} \mathrm{MD}$ doses in Japan. ${ }^{17}$

In addition, it was observed that $\mathrm{C}_{\text {max }}$ and area under the curve (AUC) of ticagrelor were observed to be $48 \%$ and $40 \%$ higher in Japanese patients compared to Caucasians, respectively. ${ }^{18}$ This observance, in accordance with the PHILO trial results of higher risk of bleeding of ticagrelor in East Asian patients, suggests that perhaps a lower dose of ticagrelor could be warranted in East Asian populations in order to minimize the adverse events while maintaining its effectiveness.

Therefore, the aim of this study was to evaluate all available $\mathrm{PK} / \mathrm{PD}$ and clinical studies involving ticagrelor at standard and reduced doses in East Asian populations to examine whether the use of lower or standard ticagrelor doses would be appropriate in this population.

\section{Methods}

\section{Search strategy}

Initially, we structured a PICO (population, intervention, comparison, and outcomes) question to guide our literature search. Our PICO question was as follows: In the East Asian population, how does low dose of ticagrelor compared with the standard dose of ticagrelor affect $\mathrm{PK} / \mathrm{PD}$ results and clinical end points?

Therefore, the literature search included terms related to ACS, East Asians, low dose, and ticagrelor (refer to Supplementary material for full search terms).

No limitations in publication dates, language, or article types were specified. We used PubMed, EMBASE, and Cochrane databases for the comprehensive literature search. The search was initially conducted in July 2016 and repeated in April 2017.

\section{Study selection}

Literature search was conducted with PubMed (84 articles), EMBASE (503 articles), and Cochrane databases (184 articles). Using the reference management program, RefWorks, 146 duplicate articles were deleted, resulting in a total of 625 articles. Two independent reviewers reviewed the titles and abstracts of the articles for the study selection process. Discrepancies regarding inclusion of articles were discussed and consensus was made with a third reviewer.

We included articles with the following characteristics: 1) prospective studies, 2) studies that compared the standard dose of ticagrelor (180 mg LD and/or $90 \mathrm{mg}$ twice daily $\mathrm{MD}$ ) to a lower LD and/or MD of ticagrelor, and 3) studies that included mostly East Asians or partially East Asians but with a subgroup analysis on Asian population. We excluded articles with the following characteristics: 1) non-clinical studies, 2) studies that did not include East Asians or those in which the percentage of East Asians included was $<10 \%$ of the total study population, and 3) studies that included only patients with special conditions (eg, reduced renal or hepatic function). Reasons for exclusion of articles are shown in Figure 1. A total of seven articles met the inclusion/exclusion criteria and were included in this analysis.

\section{Data extraction}

From each study, drug regimens, number of participants and their race, study design, inclusion/exclusion criteria, PK data $\left(\mathrm{C}_{\max }, \mathrm{AUC}, \mathrm{t}_{1 / 2}\right)$, PD data (inhibition of platelet aggregation [IPA], platelet reactivity unit [PRU]), clinical endpoints, and reported adverse events were collected when available. Data collection was performed using Microsoft Excel 2013. 


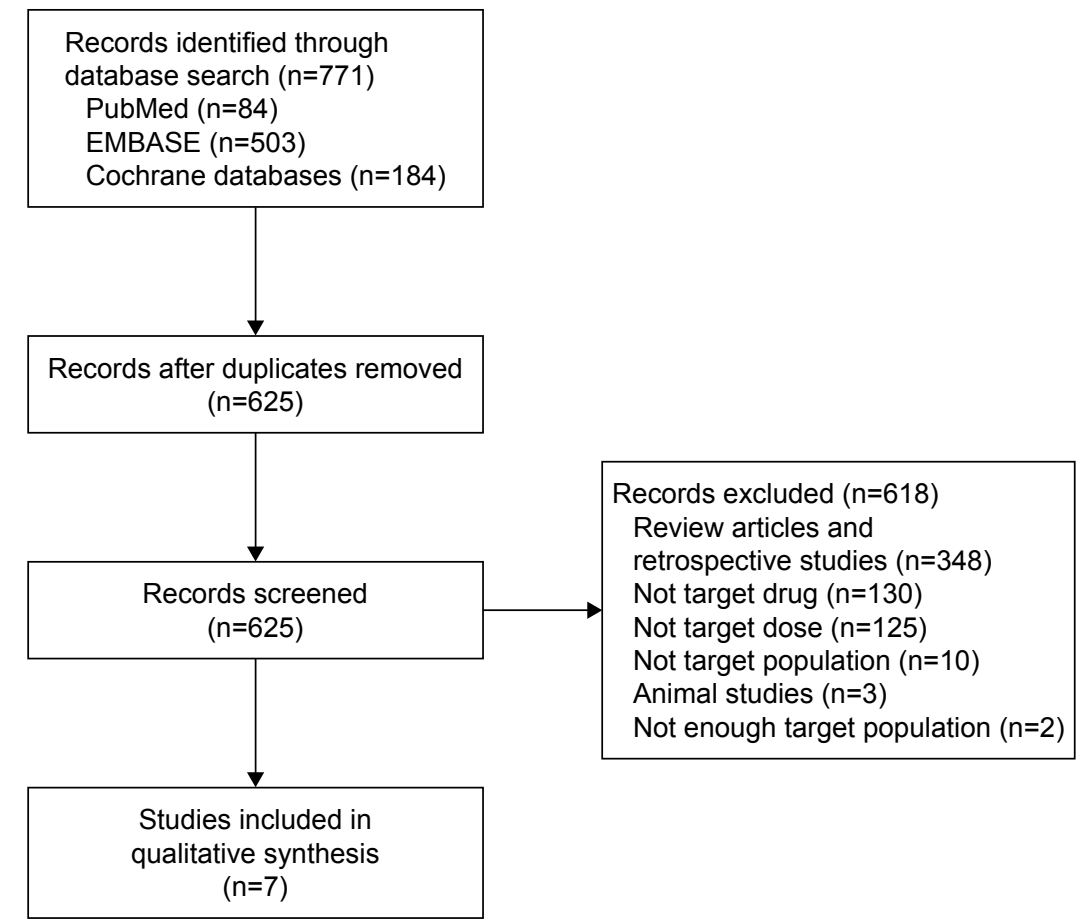

Figure I Flow diagram of the literature search and study selection process.

\section{Results}

Seven studies were included in this systematic review, which included healthy subjects, ${ }^{19,20}$ patients with ACS, ${ }^{21,22}$ patients $1-3$ years post-MI, ${ }^{1}$ and those with stable coronary artery disease. ${ }^{23,24}$ Most of the studies included the half dose of ticagrelor as the low dose (90 mg LD or $45 \mathrm{mg}$ BID MD), although one study also included the $60 \mathrm{mg}$ BID dose of ticagrelor. ${ }^{24}$ Of the seven included studies, six studies were conducted in Asia and one study was conducted worldwide including $11.0 \%$ of Asians. ${ }^{1}$ One article in Chinese was translated into English for analysis. ${ }^{21}$ All studies included ticagrelor standard-dose and lower-dose arms, and some also included placebo or clopidogrel standard-dose arms to compare the results. Participants in the study did not take any other antiplatelet or anticoagulant therapy except for the study medication and aspirin, in some cases. Table 1 summarizes the characteristics and the participants included in each study.

\section{PK data}

PK data of ticagrelor and AR-C124910XX were available in four studies. LD and MDs were evaluated in three and four of the studies, respectively, which are summarized in Table 2. In these studies, a common finding was that $\mathrm{C}_{\max }$ and AUC of ticagrelor and AR-C124910XX increased doseproportionally between $90 \mathrm{mg}$ and $180 \mathrm{mg}$ LDs, $45 \mathrm{mg}$ and
$90 \mathrm{mg}$ single doses, and $45 \mathrm{mg}$ BID and $90 \mathrm{mg}$ BID MDs. When the comparison of ticagrelor doses were twofold, the included studies showed that ticagrelor and AR-C124910XX $\mathrm{C}_{\max }$ increased 1.8-3.1 fold and 1.6-2.9 fold, respectively, and AUC increased 1.9-2.6 fold and 1.7-2.6 fold, respectively. However, in a study by Li et al ${ }^{24}$ where $45 \mathrm{mg}, 60 \mathrm{mg}$, and $90 \mathrm{mg}$ doses were compared, although there was a doseproportional increase in $\mathrm{C}_{\text {max }}$ and $\mathrm{AUC}$ between $45 \mathrm{mg}$ and $90 \mathrm{mg}$ doses, differences between ticagrelor 45 and $60 \mathrm{mg}$ were not significant.

\section{PD data}

PD data of ticagrelor were available in five studies, where final extent IPA and/or PRU were measured (Table 3). Three studies observed greater extent of platelet inhibition (higher IPA and/or lower PRU) with higher doses of ticagrelor, whereas in two studies, no significant differences in the extent of platelet inhibition (both measured by PRU) were observed between ticagrelor $45 \mathrm{mg}$ BID and $90 \mathrm{mg}$ BID doses. Four of the studies also compared the final extent IPA and/or PRU with standard dose of clopidogrel. All ticagrelor doses had considerably ${ }^{23}$ or significantly ${ }^{20-22}$ more IPA compared to clopidogrel standard dose. In addition, peak mean percentage inhibition of PRU was achieved earlier in ticagrelor $(2-4 \mathrm{~h})$ versus clopidogrel $(8 \mathrm{~h})$ group..$^{20}$ 
Table I Baseline characteristics of included trials

\begin{tabular}{|c|c|c|c|c|c|c|c|}
\hline References & Participants (n) & Race (\%) & Study design & $\begin{array}{l}\text { Study } \\
\text { type }\end{array}$ & $\begin{array}{l}\text { Study } \\
\text { duration }\end{array}$ & $\begin{array}{l}\text { Study groups } \\
\text { (n) }\end{array}$ & $\begin{array}{l}\text { Background } \\
\text { antithrombotic } \\
\text { therapy }\end{array}$ \\
\hline $\begin{array}{l}\text { Li et al, } \\
2012^{19}\end{array}$ & Healthy subjects (26) & Chinese (100\%) & $\begin{array}{l}\text { Two-cohort, } \\
\text { open-label, } \\
\text { sequential, } \\
\text { single-center } \\
\text { study }\end{array}$ & PK & $\begin{array}{l}\text { I0 days } \\
\text { (day I: single } \\
\text { dose*; days } \\
\text { 2-3: washout } \\
\text { period; days } \\
\text { 4-9: BID; day } \\
\text { I0: single dose) }\end{array}$ & $\begin{array}{l}\text { Ticagrelor } 90 \mathrm{mg} \\
(12) \\
\text { Ticagrelor } \\
180 \mathrm{mg}(14)\end{array}$ & None \\
\hline $\begin{array}{l}\text { Hiasa et al, } \\
2014^{23}\end{array}$ & $\begin{array}{l}\text { Stable CAD } \\
\text { patients (139) }\end{array}$ & $\begin{array}{l}\text { Japanese }(84.9 \%) \\
\text { Other Asians (15.1\%) }\end{array}$ & $\begin{array}{l}\text { RCT, double- } \\
\text { blinded, } \\
\text { multicenter, } \\
\text { parallel-group } \\
\text { study }\end{array}$ & PK/PD & 4 weeks & $\begin{array}{l}\text { Ticagrelor } 45 \mathrm{mg} \\
\text { BID (50) } \\
\text { Ticagrelor } 90 \mathrm{mg} \\
\text { BID (43) } \\
\text { Clopidogrel } \\
75 \text { mg daily (46) }\end{array}$ & $\begin{array}{l}\text { Aspirin } \\
75-100 \text { mg daily }\end{array}$ \\
\hline $\begin{array}{l}\text { Bonaca } \\
\text { et al, 20I5' } \\
\text { (PEGASUS- } \\
\text { TIMI 54) }\end{array}$ & $\begin{array}{l}\text { Patients I-3 years } \\
\text { post MI }(2 \mathrm{I}, \mathrm{I} 62)\end{array}$ & $\begin{array}{l}\text { European }(58.7 \%) \\
\text { North American (18.5\%) } \\
\text { South American (II.6\%) } \\
\text { Asian (II.0\%) }\end{array}$ & $\begin{array}{l}\text { RCT, ITT, } \\
\text { double-blinded, } \\
\text { double-dummy, } \\
\text { multicenter, } \\
\text { multinational, } \\
\text { parallel-group } \\
\text { study }\end{array}$ & $\begin{array}{l}\text { Clinical } \\
\text { study }\end{array}$ & $\begin{array}{l}\text { Median } \\
\text { follow-up of } \\
33 \text { months }\end{array}$ & $\begin{array}{l}\text { Ticagrelor } 60 \mathrm{mg} \\
\text { BID }(7,045) \\
\text { Ticagrelor } 90 \mathrm{mg} \\
\text { BID }(7,050) \\
\text { Placebo }(7,067)\end{array}$ & $\begin{array}{l}\text { Aspirin } \\
75-150 \mathrm{mg} \text { daily }\end{array}$ \\
\hline $\begin{array}{l}\text { Li et al, } \\
2015^{21}\end{array}$ & $\begin{array}{l}\text { Patients with unstable } \\
\text { angina pectoris and } \\
\text { non-revascularization } \\
(35 \mathrm{I})\end{array}$ & Chinese (100\%) & $\begin{array}{l}\mathrm{RCT}, \\
\text { parallel-group } \\
\text { study }\end{array}$ & $\begin{array}{l}\text { PD/clinical } \\
\text { study }\end{array}$ & $\begin{array}{l}\text { Follow-up of } \\
12 \text { months }\end{array}$ & $\begin{array}{l}\text { Ticagrelor } 90 \mathrm{mg} \\
\text { LD/45 mg BID } \\
\text { MD (I08) } \\
\text { Ticagrelor } \\
\text { I } 80 \text { mg LD/90 mg } \\
\text { BID MD (I32) } \\
\text { Clopidogrel } \\
300 \text { mg LD/75 mg } \\
\text { daily MD (III) }\end{array}$ & $\begin{array}{l}\text { Aspirin } 100 \mathrm{mg} \\
\text { daily }\end{array}$ \\
\hline $\begin{array}{l}\text { Li et al, } \\
2016^{24}\end{array}$ & $\begin{array}{l}\text { Stable CAD } \\
\text { patients (36) }\end{array}$ & Chinese (100\%) & $\begin{array}{l}\text { RCT, open-label, } \\
\text { single-center } \\
\text { study }\end{array}$ & PK/PD & $\begin{array}{l}7 \text { days } \\
\text { (day I: single } \\
\text { dose; days } \\
\text { 3-6: BID; day } \\
\text { 7: single dose) }\end{array}$ & $\begin{array}{l}\text { Ticagrelor } 45 \mathrm{mg} \\
(\mathrm{I} 2) \\
\text { Ticagrelor } 60 \mathrm{mg} \\
\text { (I2) } \\
\text { Ticagrelor } 90 \mathrm{mg} \\
(\mathrm{I} 2)\end{array}$ & $\begin{array}{l}\text { Aspirin } \\
75-100 \text { mg daily }\end{array}$ \\
\hline $\begin{array}{l}\text { Li et al, } \\
2016^{20}\end{array}$ & Healthy subjects $(30)$ & Chinese (100\%) & $\begin{array}{l}\text { RCT, open-label, } \\
\text { single-center } \\
\text { study }\end{array}$ & PK/PD & 4 days & $\begin{array}{l}\text { Ticagrelor } 90 \mathrm{mg} \\
\text { LD/45 mg BID } \\
\text { MD (I0) } \\
\text { Ticagrelor } \\
\text { I80 mg LD/90 mg } \\
\text { BID MD (I0) } \\
\text { Clopidogrel } \\
600 \text { mg LD/75 mg } \\
\text { daily MD (10) }\end{array}$ & None \\
\hline $\begin{array}{l}\text { Xue et al, } \\
2016^{22}\end{array}$ & $\begin{array}{l}\text { NSTE-ACS } \\
\text { patients (6I) }\end{array}$ & Chinese (100\%) & $\begin{array}{l}\text { RCT, double- } \\
\text { blinded, single- } \\
\text { center, parallel- } \\
\text { group study }\end{array}$ & PD & 5 days & $\begin{array}{l}\text { Ticagrelor } 90 \mathrm{mg} \\
\text { LD/45 mg BID } \\
\text { MD (20) } \\
\text { Ticagrelor } \\
\text { I } 80 \text { mg LD/90 mg } \\
\text { BID MD (2I) } \\
\text { Clopidogrel } \\
300 \text { mg LD/75 mg } \\
\text { daily MD (20) }\end{array}$ & Aspirin daily \\
\hline
\end{tabular}

Note: *For the purposes of this review, only the results of the single dose study were included.

Abbreviations: CAD, coronary artery disease; ITT, intention to treat; LD, loading dose; MD, maintenance dose; MI, myocardial infarction; NSTE-ACS, non-ST segment elevation acute coronary syndrome; PK, pharmacokinetics; PD, pharmacodynamics; RCT, randomized controlled trial. 
Table 2 Pharmacokinetic data of low dose versus standard dose of ticagrelor in East Asians

\begin{tabular}{|c|c|c|c|c|c|c|c|c|c|}
\hline \multirow[t]{2}{*}{ References } & \multirow{2}{*}{$\begin{array}{l}\text { Ticagrelor } \\
\text { dose }\end{array}$} & \multirow[t]{2}{*}{ Time } & \multicolumn{3}{|c|}{ Ticagrelor } & \multicolumn{3}{|c|}{ AR-CI249I0XX } & \multirow[t]{2}{*}{ Main findings } \\
\hline & & & $\begin{array}{l}C_{\max } \\
(\mathrm{ng} / \mathrm{mL})\end{array}$ & $\begin{array}{l}\text { AUC }_{0 \text {-inf }} \\
(\mathrm{ng} \cdot \mathrm{h} / \mathrm{mL})\end{array}$ & $t^{1} 1 / 2(h)$ & $\begin{array}{l}C_{\max } \\
(n g / m L)\end{array}$ & $\begin{array}{l}\text { AUC }_{0 \text {-inf }} \\
(\mathrm{ng} \cdot \mathrm{h} / \mathrm{mL})\end{array}$ & $t^{\prime} / 2(h)$ & \\
\hline \multirow{4}{*}{$\begin{array}{l}\text { Li et al, } \\
2012^{19}\end{array}$} & $90 \mathrm{mg}$ & Day I (LD) & $602(32)$ & $3,855(3 \mathrm{I})$ & $10.9(36)$ & $183(25)$ & $\mathrm{I}, 797(25)$ & $9.5(29)$ & \multirow{2}{*}{$\begin{array}{l}\mathrm{C}_{\text {max }} \text { and } \mathrm{AUC}_{\text {0-inf }} \\
\text { of ticagrelor and }\end{array}$} \\
\hline & $180 \mathrm{mg}$ & & $1,198(30)$ & $7,738(33)$ & $11.7(28)$ & $345(25)$ & $2,997(25)$ & $9.1(15)$ & \\
\hline & & & & & & & & & AR-CI249I0XX increased \\
\hline & $45 \mathrm{mg}$ & Day I (LD) & $283(5 \mathrm{I})$ & I,640 (46) \# & $5.10(27)$ & $62.8(48)$ & $445(39)^{\#}$ & $8.16(38)$ & \multirow{4}{*}{$\begin{array}{l}\text { dose-proportionally } \\
\mathrm{C}_{\text {max }} \text { and } A U C_{0-t} \\
\text { of ticagrelor and } \\
\text { AR-CI249IOXX increased } \\
\text { dose-proportionally in both } \\
\text { Japanese and non-Japanese }\end{array}$} \\
\hline \multirow{4}{*}{$\begin{array}{l}\text { Hiasa et al, } \\
2014^{23, *}\end{array}$} & $90 \mathrm{mg}$ & Day 28 (MD) & $612(39)$ & $3,310(36)^{\#}$ & $4.83(27)$ & $156(45)$ & $1,080(30)^{\#}$ & $7.13(43)$ & \\
\hline & $45 \mathrm{mg}$ & & $422(50)$ & $3,050(55)^{\#}$ & $9.72(29)$ & $135(40)$ & $\mathrm{I}, 180(42)^{\#}$ & $15.57(36)$ & \\
\hline & $90 \mathrm{mg}$ & & 931 (43) & $6,080(4 I)^{\#}$ & $8.78(22)$ & $326(36)$ & $2,720(31)^{\#}$ & $|4.4|(30)$ & \\
\hline & & & & & & & & & \multirow{3}{*}{$\begin{array}{l}\text { Asian patients } \\
\mathrm{C}_{\text {max }} \text { and } A \cup C_{0-12} \\
\text { of ticagrelor and }\end{array}$} \\
\hline \multirow{6}{*}{$\begin{array}{l}\text { Li et al, } \\
2016^{24}\end{array}$} & $45 \mathrm{mg}$ & Day I (LD) & $464(38)$ & $3,220(5 \mathrm{I})$ & $10.72(16.18)$ & $88.3(24.6)$ & $922(29)$ & $12.65(22.94)$ & \\
\hline & $60 \mathrm{mg}$ & & $414(34)$ & $3,633(32)$ & $9.42(13.53)$ & $77.1(54)$ & $1,108(35)$ & II.38 (24.27) & \\
\hline & $90 \mathrm{mg}$ & & $822(37)$ & $6,234(54)$ & $10.14(17.54)$ & $139(38)$ & $\mathrm{I}, 644(3 \mathrm{I})$ & II.62 (24.64) & AR-CI24910XX increased \\
\hline & $45 \mathrm{mg}$ & Day 7 (MD) & $616(37)$ & $3,882(42)^{\ddagger}$ & N/A & $144(26)$ & $1,069(25)^{\ddagger}$ & N/A & dose-proportionally \\
\hline & $60 \mathrm{mg}$ & & $689(34)$ & 4,35 I $(37)^{\ddagger}$ & $N / A$ & $180(50)$ & $\mathrm{I}, 3 \mid 4(4 \mathrm{I})^{\ddagger}$ & $\mathrm{N} / \mathrm{A}$ & between ticagrelor \\
\hline & $90 \mathrm{mg}$ & & $\mathrm{I}, 273(43)$ & $8,206(5 I)^{\ddagger}$ & $N / A$ & $30 \mathrm{I}(32)$ & $2,254(37)^{\ddagger}$ & $\mathrm{N} / \mathrm{A}$ & $\begin{array}{l}45 \mathrm{mg} \text { and } 90 \mathrm{mg} \text { doses, } \\
\text { but differences between } \\
\text { ticagrelor } 45 \mathrm{mg} \text { and } 60 \mathrm{mg} \\
\text { doses were not significant }\end{array}$ \\
\hline Li et al, & $90 \mathrm{mg}$ & Day I (LD) & $470(48)$ & $3,330(22)$ & $N / A$ & I54 (39) & $887(25)^{\#}$ & $\mathrm{~N} / \mathrm{A}$ & $C_{\max }$ and $A \cup C_{0 \text {-inf }}$ \\
\hline \multirow[t]{3}{*}{$2016^{20}$} & $180 \mathrm{mg}$ & Day 4 (MD) & $\mathrm{I}, 447(27)$ & $6,905(30)$ & $N / A$ & $445(35)$ & $2,120(34)^{\#}$ & $\mathrm{~N} / \mathrm{A}$ & of ticagrelor and \\
\hline & $45 \mathrm{mg}$ & & $322(18)$ & $3,128(18)$ & $8.4(37)$ & $142(35)$ & $2,467(33)$ & $16.6(54)$ & \multirow{2}{*}{$\begin{array}{l}\text { AR-CI24910XX increased } \\
\text { dose-proportionally }\end{array}$} \\
\hline & $90 \mathrm{mg}$ & & $921(46)$ & $8,023(55)$ & $8.9(31)$ & $361(35)$ & $6,407(46)$ & II.7 (32) & \\
\hline
\end{tabular}

Notes: All data are provided as geometric mean (coefficient of variation \%) unless otherwise indicated. *lapanese patient data ( $\mathrm{n}=38$ ); ${ }^{\#} \mathrm{AUC} \mathrm{C}_{0-\mathrm{t}}(\mathrm{AUC}$ from time 0 to end of the dosing interval); ${ }^{\ddagger} \mathrm{UUC}_{0-12}$.

Abbreviations: LD, loading dose; MD, maintenance dose; N/A, not available; PK, pharmacokinetics; AUC, area under the curve.

\section{Clinical outcomes: efficacy}

Clinical efficacy outcomes were evaluated in two studies (Table 4). ${ }^{21,25}$ PEGASUS-TIMI 54 is a clinical trial that compared the efficacy and safety of ticagrelor $60 \mathrm{mg}$ BID, $90 \mathrm{mg}$ BID, and placebo in a total of 21,162 patients who are 1-3 years post-MI for a median follow-up of 33 months. ${ }^{25}$ Of these, $11.0 \%$ of the patients were from Asia. The primary efficacy endpoint of this study was the composite of $\mathrm{CV}$ death, MI, or stroke. In ticagrelor $60 \mathrm{mg}, 90 \mathrm{mg}$, and placebo group, this primary efficacy endpoint occurred in $7.77 \%$, $7.85 \%$, and $9.04 \%$ of the patients. Both of the ticagrelor groups had significantly less events compared to placebo, but significant differences in the efficacy between the two doses were not observed. When the primary efficacy outcome was analyzed by race and by region, interaction $p$-value was not statistically significant, indicating that the efficacy outcome was not statistically influenced by race.

Li et $\mathrm{al}^{21}$ evaluated the efficacy outcomes in 351 Chinese patients with unstable angina pectoris and nonrevascularization. Patients were given either standard-dose or low-dose (90 mg LD/45 mg BID MD) ticagrelor for 12 months. They evaluated the rates of CV death, MI, stroke, PCI, and recurrent angina and found that there were no significant differences in the rates between the ticagrelor doses for all outcomes measured.

\section{Clinical outcomes: safety}

All seven studies reported the safety and tolerability data (Table 5). In studies that lasted for less than 7 days, no major bleeding was reported. ${ }^{19,20,22,24}$ In a study by Li et al, ${ }^{19}$ one case of minor bleeding was observed in each of the ticagrelor $90 \mathrm{mg}$ and $180 \mathrm{mg}$ group. In another study by $\mathrm{Li}$ et al, ${ }^{24}$ ticagrelor $45 \mathrm{mg}, 60 \mathrm{mg}$, and $90 \mathrm{mg}$ had one (8.3\%), two (16.7\%), and one (8.3\%) patients with minor bleeding, respectively. In a study by Xue et $\mathrm{al},{ }^{22}$ minor bleeding was observed only in ticagrelor $90 \mathrm{mg}$ group $(9.5 \%)$. In a study by $\mathrm{Li}$ et al, ${ }^{20}$ minimal bleeding was reported in one and two patients in ticagrelor low-dose and standard-dose groups, respectively, whereas no cases of minimal bleeding were observed in the clopidogrel group.

Both major and minor bleeding were observed in studies over 4 weeks of duration. ${ }^{1,21,23}$ In a study by Hiasa et al, ${ }^{23}$ stable CAD patients were given ticagrelor $45 \mathrm{mg}$ BID, $90 \mathrm{mg}$ BID, or clopidogrel $75 \mathrm{mg}$ daily for 4 weeks with underlying low-dose aspirin therapy. Only one patient in the low-dose ticagrelor group reported major bleeding. Regarding minimal 
Table 3 Pharmacodynamic data of low dose versus standard dose ticagrelor in East Asians

\begin{tabular}{|c|c|c|c|c|c|}
\hline \multirow[t]{2}{*}{ References } & \multirow[t]{2}{*}{ Treatment } & \multirow[t]{2}{*}{ Time } & \multicolumn{2}{|l|}{ PD } & \multirow[t]{2}{*}{ Main findings } \\
\hline & & & $\begin{array}{l}\text { Final } \\
\text { extent IPA }\end{array}$ & PRU & \\
\hline $\begin{array}{l}\text { Hiasa et al, } \\
2014^{23}\end{array}$ & $\begin{array}{l}\text { Ticagrelor } 45 \mathrm{mg} \\
\text { Ticagrelor } 90 \mathrm{mg} \\
\text { Clopidogrel } 75 \mathrm{mg}\end{array}$ & Day 28 & $\begin{array}{l}57 \% \\
67 \% \\
42 \%\end{array}$ & $\mathrm{~N} / \mathrm{A}$ & $\begin{array}{l}\text { Final extent IPA was greater at every time point on } \\
\text { days I and } 28 \text { with ticagrelor } 90 \mathrm{mg} \text { than } 45 \mathrm{mg} \text {. } \\
\text { Both doses of ticagrelor had greater final extent } \\
\text { IPA than clopidogrel } 75 \mathrm{mg} \text { at all time points }\end{array}$ \\
\hline Li et al, $2015^{21}$ & $\begin{array}{l}\text { Ticagrelor } 45 \mathrm{mg} \\
\text { Ticagrelor } 90 \mathrm{mg} \\
\text { Clopidogrel } 75 \mathrm{mg}\end{array}$ & Days I-7 & $\begin{array}{l}\text { N/A } \\
\text { N/A } \\
\text { N/A }\end{array}$ & $\begin{array}{l}\text { N/A } \\
\text { N/A } \\
\text { N/A }\end{array}$ & $\begin{array}{l}\text { IPA of ticagrelor } 90 \mathrm{mg} \text { was higher than ticagrelor } \\
45 \mathrm{mg} \text {, which was higher than clopidogrel } 75 \mathrm{mg} \text {. } \\
\text { PRU and PRI of ticagrelor } 90 \mathrm{mg} \text { were lower than } \\
\text { ticagrelor } 45 \mathrm{mg} \text {, which was lower than clopidogrel } \\
75 \mathrm{mg} \text {. All differences were statistically significant }\end{array}$ \\
\hline Li et al, $2016^{24}$ & $\begin{array}{l}\text { Ticagrelor } 45 \mathrm{mg} \\
\text { Ticagrelor } 60 \mathrm{mg} \\
\text { Ticagrelor } 90 \mathrm{mg} \\
\text { Ticagrelor } 45 \mathrm{mg} \\
\text { Ticagrelor } 60 \mathrm{mg} \\
\text { Ticagrelor } 90 \mathrm{mg}\end{array}$ & Day 7 & $\begin{array}{l}27 \%(26) \\
26 \%(26) \\
33 \%(31) \\
>85 \% \\
>90 \% \\
>95 \%\end{array}$ & $\begin{array}{l}N / A \\
N / A \\
N / A \\
48-98 \\
36-8 \mid \\
\mid 7-32\end{array}$ & $\begin{array}{l}\text { IPA increased dose-dependently. Reductions in } \\
\text { mean PRU values were dose dependent }\end{array}$ \\
\hline Li et al, $2016^{20}$ & $\begin{array}{l}\text { Ticagrelor } 45 \mathrm{mg} \\
\text { Ticagrelor } 90 \mathrm{mg} \\
\text { Clopidogrel } 75 \mathrm{mg}\end{array}$ & Days I-4 & $\begin{array}{l}\text { N/A } \\
\text { N/A } \\
\text { N/A }\end{array}$ & $\begin{array}{l}97.1 \% * \\
98.7 \% * \\
77 \% *\end{array}$ & $\begin{array}{l}\text { No significant differences in PRU were observed } \\
\text { between low- and standard-dose ticagrelor, but } \\
\text { both doses of ticagrelor were significantly higher } \\
\text { than clopidogrel at } 0.5, I, 2,4,8 \text {, and } 48 \mathrm{~h} \text { after } \\
\text { loading and maintenance doses }(p<0.05)\end{array}$ \\
\hline $\begin{array}{l}\text { Xue et al, } \\
2016^{22}\end{array}$ & $\begin{array}{l}\text { Ticagrelor } 45 \mathrm{mg} \\
\text { Ticagrelor } 90 \mathrm{mg} \\
\text { Clopidogrel } 75 \mathrm{mg}\end{array}$ & Day 5 & $\begin{array}{l}\text { N/A } \\
\text { N/A } \\
\text { N/A }\end{array}$ & $\begin{array}{l}44.55 \pm 32.88^{\#} \\
39.10 \pm 40.02^{\#} \\
189.20 \pm 65.22^{\#}\end{array}$ & $\begin{array}{l}\text { PRU of ticagrelor } 45 \mathrm{mg} \text { was similar to ticagrelor } \\
90 \mathrm{mg}(p=0.3085) \text {, but both doses of ticagrelor had } \\
\text { significantly lower PRU than clopidogrel }(p<0.000 \text { I) }\end{array}$ \\
\hline
\end{tabular}

Notes: *Peak mean percentage inhibition of PRU; ${ }^{*}$ mean $\pm \mathrm{SD}$.

Abbreviations: IPA, inhibition of platelet aggregation; N/A, not available; PD, pharmacodynamics; PRI, platelet reactivity index; PRU, P2Y 12 reactivity unit.

bleeding, ticagrelor $45 \mathrm{mg}$ BID had 26.0\%, $90 \mathrm{mg}$ BID had $39.5 \%$, and clopidogrel group had $21.7 \%$ incidence. In PEGASUS-TIMI 54, where patients were followed up for a median of 33 months, $2.3 \%$ of patients in ticagrelor
$60 \mathrm{mg}$ BID group and 2.6\% in ticagrelor $90 \mathrm{mg}$ BID group had major bleeding, which were both significantly more compared to the placebo group (1.1\%), but statistical significance between the two ticagrelor doses were not reported. When the

Table 4 Clinical efficacy outcomes of low-dose versus standard-dose ticagrelor in East Asians

\begin{tabular}{|c|c|c|c|}
\hline \multirow{3}{*}{$\begin{array}{l}\text { References } \\
\text { Bonaca et al, 20I5' } \\
\text { (PEGASUS-TIMI 54) }\end{array}$} & \multirow{2}{*}{$\begin{array}{l}\text { Participants } \\
\text { All patients }\end{array}$} & \multicolumn{2}{|c|}{ Clinical efficacy outcomes } \\
\hline & & Composite of & Ticagrelor 60 mg 7.77\%, HR* 0.84 (95\% Cl 0.74-0.95) \\
\hline & & cardiovascular & Ticagrelor 90 mg 7.85\%, HR* 0.85 (95\% Cl 0.75-0.96) \\
\hline & & death, Ml, or stroke & Placebo $9.04 \%$ \\
\hline & Asians only & Composite of & Ticagrelor $60 \mathrm{mg} 7.11 \%, \mathrm{HR} * 0.90(95 \% \mathrm{Cl} 0.60-1.34) p=0.83^{\ddagger}$ \\
\hline & & cardiovascular & Ticagrelor $90 \mathrm{mg} 6.08 \%, \mathrm{HR}^{*} 0.82(95 \% \mathrm{Cl} 0.54-1.23) p=0.99^{\ddagger}$ \\
\hline \multirow{11}{*}{ Li et al, $2015^{21^{\#}}$} & & death, MI, or stroke & Placebo $6.86 \%$ \\
\hline & (Chinese) & CV death & Low-dose ticagrelor $2.8 \%$ \\
\hline & & & Standard-dose ticagrelor $2.3 \%, \mathrm{HR}$ I.I $08(95 \% \mathrm{Cl} 0.974-1.259), p=0.118$ \\
\hline & & MI & Low-dose ticagrelor $3.7 \%$ \\
\hline & & & Standard-dose ticagrelor $3.9 \%, \mathrm{HR}$ I. $108(95 \% \mathrm{Cl} 0.974-1.259), p=0.118$ \\
\hline & & Stroke & Low-dose ticagrelor $0.9 \%$ \\
\hline & & & Standard-dose ticagrelor $0.8 \%$, HR I. $106(95 \% \mathrm{Cl} 0.974-1.257), p=0.12 \mathrm{I}$ \\
\hline & & $\mathrm{PCl}$ & Low-dose ticagrelor $1.9 \%$ \\
\hline & & & Standard-dose ticagrelor I.5\%, HR I.I $07(95 \% \mathrm{Cl} 0.974-1.258), p=0.120$ \\
\hline & & Recurrent angina & Low-dose ticagrelor $7.4 \%$ \\
\hline & & & Standard-dose ticagrelor $6.8 \%, \mathrm{HR}$ I. $109(95 \% \mathrm{Cl} 0.973-1.264), p=0.119$ \\
\hline
\end{tabular}

Notes: *Compared to placebo; " low-dose ticagrelor: $90 \mathrm{mg}$ LD/45 mg BID MD and standard-dose ticagrelor: $180 \mathrm{mg}$ LD/90 mg BID MD; ;interaction p-value (for region). Abbreviations: $\mathrm{Cl}$, confidence interval; $\mathrm{CV}$ death, cardiovascular death; $\mathrm{HR}$, hazard ratio; $\mathrm{PCl}$, percutaneous coronary intervention; MI, myocardial infarction; LD, loading dose; MD, maintenance dose. 
Table 5 Safety and tolerability of low-dose versus standard-dose ticagrelor in East Asians

\begin{tabular}{|c|c|c|c|c|c|}
\hline References & & Major bleeding (\%) & $\begin{array}{l}\text { Minor/minimal } \\
\text { bleeding (\%) }\end{array}$ & Dyspnea (\%) & $\begin{array}{l}\text { Any adverse events } \\
\text { (\%) }\end{array}$ \\
\hline Li et al, $2012^{19}$ & & None & $\begin{array}{l}\text { Minor bleeding: } \\
\text { Ticagrelor } 90 \text { mg 8.3\% } \\
\text { (epistaxis) } \\
\text { Ticagrelor } 180 \mathrm{mg} 7.1 \% \\
\text { (gingival bleeding) }\end{array}$ & $\begin{array}{l}\text { Ticagrelor } 90 \mathrm{mg} 0 \% \\
\text { Ticagrelor } 180 \mathrm{mg} \\
21.4 \%\end{array}$ & $\begin{array}{l}\text { Ticagrelor } 90 \mathrm{mg} 25.0 \% \\
\text { Ticagrelor } 180 \mathrm{mg} \\
50.0 \%\end{array}$ \\
\hline Hiasa et al, $2014^{23}$ & & $\begin{array}{l}\text { Ticagrelor } 45 \mathrm{mg} 2 \% \\
\text { Ticagrelor } 90 \mathrm{mg} 0 \% \\
\text { Clopidogrel } 75 \mathrm{mg} 0 \%\end{array}$ & $\begin{array}{l}\text { Minor bleeding: None } \\
\text { Minimal bleeding: } \\
\text { Ticagrelor } 45 \mathrm{mg} 26.0 \% \\
\text { Ticagrelor } 90 \mathrm{mg} 39.5 \% \\
\text { Clopidogrel } 75 \mathrm{mg} 21.7 \%\end{array}$ & $\begin{array}{l}\text { Ticagrelor } 45 \mathrm{mg} 0 \% \\
\text { Ticagrelor } 90 \mathrm{mg} 4.7 \% \\
\text { Clopidogrel } 75 \mathrm{mg} 0 \%\end{array}$ & $\begin{array}{l}\text { Ticagrelor } 45 \mathrm{mg} 56.0 \% \\
\text { Ticagrelor } 90 \mathrm{mg} 74.4 \% \\
\text { Clopidogrel } 75 \mathrm{mg} \\
47.8 \%\end{array}$ \\
\hline $\begin{array}{l}\text { Bonaca et al, } 2015^{\prime} \\
\text { (PEGASUS-TIMI 54) }\end{array}$ & All patients & $\begin{array}{l}\text { Ticagrelor } 60 \mathrm{mg} 2.3 \% \text {, } \\
\mathrm{HR}^{*} 2.32(95 \% \mathrm{Cl} \\
\text { I.68-3.2I) } \\
\text { Ticagrelor } 90 \mathrm{mg} 2.6 \% \text {, } \\
\mathrm{HR}^{*} 2.69(95 \% \mathrm{Cl} \\
\text { I.96-3.70) } \\
\text { Placebo I.1\% }\end{array}$ & 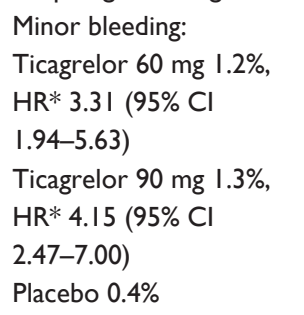 & $\begin{array}{l}\text { Ticagrelor } 60 \mathrm{mg} 15.8 \% \\
\text { Ticagrelor } 90 \mathrm{mg} 18.9 \% \\
\text { Placebo } 6.4 \%\end{array}$ & $\begin{array}{l}\text { Ticagrelor } 60 \mathrm{mg} 76.3 \% \\
\text { Ticagrelor } 90 \mathrm{mg} 76.9 \% \\
\text { Placebo } 70.0 \%\end{array}$ \\
\hline & Asians only & $\begin{array}{l}\text { Ticagrelor } 60 \mathrm{mg} 3.7 \% \text {, } \\
\mathrm{HR}^{*} 3.23(95 \% \mathrm{Cl} \\
\text { I.45-7.18) } \\
\text { Ticagrelor } 90 \mathrm{mg} 3.0 \% \text {, } \\
\mathrm{HR}^{*} 2.37(95 \% \mathrm{Cl} \\
\text { I.02-5.50) } \\
\text { Placebo I.4\% }\end{array}$ & N/A & $\mathrm{N} / \mathrm{A}$ & N/A \\
\hline Li et al, $2015^{21}$ & & $\begin{array}{l}\text { Ticagrelor } 45 \mathrm{mg} 4.6 \% \text {, } \\
\mathrm{HR}^{\#} 0.352(95 \% \mathrm{Cl} \\
0.125-0.994) \\
\text { Ticagrelor } 90 \mathrm{mg} 12.1 \% \\
\text { Clopidogrel } 75 \mathrm{mg} \\
3.6 \%\end{array}$ & $\begin{array}{l}\text { Minor bleeding: } \\
\text { Ticagrelor } 45 \mathrm{mg} 5.6 \% \text {, } \\
\mathrm{HR}^{\#} 0.3 \mathrm{II}(95 \% \mathrm{Cl} \\
0.12 \mathrm{I}-0.80 \mathrm{I}) \\
\text { Ticagrelor } 90 \mathrm{mg} 15.9 \% \\
\text { Clopidogrel } 75 \mathrm{mg} 4.5 \%\end{array}$ & $\begin{array}{l}\text { Ticagrelor } 45 \mathrm{mg} 3.7 \% \\
\text { Ticagrelor } 90 \mathrm{mg} \text { I I.4\% } \\
\text { HR I.086 ( } 95 \% \mathrm{Cl} \\
0.952-1.237) \\
\text { Clopidogrel } 75 \mathrm{mg} \mathrm{N} / \mathrm{A}\end{array}$ & $\mathrm{N} / \mathrm{A}$ \\
\hline Li et al, $2016^{24}$ & & None & $\begin{array}{l}\text { Minor bleeding: } \\
\text { Ticagrelor } 45 \text { mg 8.3\% } \\
\text { Ticagrelor } 60 \mathrm{mg} \mathrm{16.7 \%} \\
\text { Ticagrelor } 90 \mathrm{mg} 8.3 \%\end{array}$ & $\begin{array}{l}\text { Ticagrelor } 45 \mathrm{mg} 0 \% \\
\text { Ticagrelor } 60 \mathrm{mg} 16.7 \% \\
\text { Ticagrelor } 90 \mathrm{mg} 8.3 \%\end{array}$ & $\begin{array}{l}\text { Ticagrelor } 45 \mathrm{mg} 33.3 \% \\
\text { Ticagrelor } 60 \mathrm{mg} 33.3 \% \\
\text { Ticagrelor } 90 \mathrm{mg} 41.7 \%\end{array}$ \\
\hline Li et al, $2016^{20}$ & & None & $\begin{array}{l}\text { Minimal bleeding: } \\
\text { Ticagrelor } 90 \text { mg LD, } \\
45 \text { mg BID MD I0.0\% } \\
\text { Ticagrelor } 180 \text { mg LD, } \\
90 \text { mg BID MD } 20.0 \% \\
\text { Clopidogrel } 600 \text { mg LD, } \\
75 \text { mg daily MD 0\% }\end{array}$ & None & $\begin{array}{l}\text { Ticagrelor groups N/A } \\
\text { Clopidogrel } \\
600 \mathrm{mg} \text { LD, } 75 \mathrm{mg} \\
\text { daily MD } 10.0 \% \\
\text { (sinus bradycardia) }\end{array}$ \\
\hline Xue et al, $2016^{22}$ & & None & $\begin{array}{l}\text { Minor bleeding: } \\
\text { Ticagrelor } 45 \mathrm{mg} \mathrm{0 \%} \\
\text { Ticagrelor } 90 \mathrm{mg} 9.5 \% \\
\text { Clopidogrel } 75 \mathrm{mg} 0 \%\end{array}$ & $\begin{array}{l}\text { Ticagrelor } 45 \mathrm{mg} \text { 10.0\% } \\
\text { Ticagrelor } 90 \mathrm{mg} 14.3 \% \\
\text { Clopidogrel } 75 \text { mg } 0 \%\end{array}$ & $\begin{array}{l}\text { Ticagrelor } 45 \mathrm{mg} \text { 15.0\% } \\
\text { Ticagrelor } 90 \mathrm{mg} 33.3 \% \\
\text { Clopidogrel } 75 \text { mg 0\% }\end{array}$ \\
\hline
\end{tabular}

Notes: *Compared to placebo; ${ }^{*}$ compared to ticagrelor $90 \mathrm{mg}$; bleeding events compared to clopidogrel were not statistically significant $(p>0.05) ;{ }^{*}$ composite of several minor bleeding results (positive occult bleeding, hemoptysis, gingival bleeding, epistaxis).

Abbreviations: $\mathrm{Cl}$, confidence interval; HR, hazard ratio; N/A, not available; TIMI, thrombolysis in myocardial infarction; LD, loading dose; MD, maintenance dose.

subgroup of patients from Asia was analyzed, similar results were observed, with the exception of ticagrelor $60 \mathrm{mg}$ BID dose having a higher numerical incidence (3.7\%) of major bleeding than ticagrelor $90 \mathrm{mg}$ BID dose (3.0\%). ${ }^{1}$ In a study by Li et al, ${ }^{21}$ patients were followed up for 12 months and major bleeding occurred in $4.6 \%$ and $12.1 \%$ of patients in the low- and standard-dose ticagrelor groups, respectively, and this difference was statistically significant. Patients in the clopidogrel group had 3.6\% incidence of major bleeding, and this rate was not statistically different from the incidence 
observed for low-dose ticagrelor group. Regarding minor bleeding, the low-dose ticagrelor group (5.6\%) and clopidogrel group (4.5\%) had similar incidence, but standard-dose ticagrelor group had statistically more incidence of minor bleeding (15.9\%).

Dyspnea was reported in most of the studies with a trend of increasing incidence with increasing dose, but one study that compared ticagrelor $45 \mathrm{mg}$ (3.7\%) to $90 \mathrm{mg}$ (11.4\%) found no statistical significance between the two groups (hazards ratio [HR] 1.086, 95\% confidence interval [95\% CI] 0.952-1.237). ${ }^{21}$ Incidence of any adverse events were reported, but its statistical significance is difficult to determine as in some studies, the incidence was numerically similar between ticagrelor doses, but in other cases, more adverse event rates were reported in standard dose of ticagrelor. No statistical analysis on any adverse events was conducted in the seven included studies.

\section{Discussion}

Ticagrelor is a potent $\mathrm{P} 2 \mathrm{Y}_{12}$ receptor antagonist used for the prevention of recurrent ACS in combination with aspirin. Ticagrelor has more potent and consistent antiplatelet effects than clopidogrel, a widely used $\mathrm{P} 2 \mathrm{Y}_{12}$ receptor antagonist. Clopidogrel is a prodrug that requires two-step metabolism by the CYP enzymes to become an active moiety, and this results in large interpersonal variations in clopidogrel drug response contributory to CYP enzyme polymorphisms. ${ }^{26,27}$

However, in the PHILO study conducted in East Asians, ticagrelor did not demonstrate superior efficacy over clopidogrel and had a trend toward higher major bleeding (HR 1.54, 95\% CI 0.94-2.53) and a significantly higher rate of combined major and minor bleeding (HR 1.72, 95\% CI 1.23-2.40). ${ }^{8}$ Many limitations of PHILO exist, including small sample size, differences in baseline characteristics, early trial closure, and application of questionable statistics. ${ }^{9}$ Despite many shortcomings, the findings of this study suggest a need to evaluate the lower dose of ticagrelor in East Asians, in the observance of increased bleeding compared to clopidogrel.

Maintaining an adequate antiplatelet efficacy while minimizing the risk of adverse events is crucial to the successful treatment of $\mathrm{P} 2 \mathrm{Y}_{12}$ receptor antagonists. It has been suggested that East Asian patients seem to have a different therapeutic window for platelet reactivity; while East Asians tend to result in high platelet reactivity after administration of antiplatelet agent(s), they tend to result in lower thrombotic events, which is referred to as the "East Asian paradox". 29,30 Rationale behind such disparity of responses in East Asians compared to other races is not entirely clear, but it may be attributable to several factors, such as genetic differences and lower levels of hemostatic factors, endothelial markers, and inflammatory markers in East Asian population, which are known to be associated with risk of CV diseases. ${ }^{30-32}$

Increased risk of bleeding in East Asians has been observed, where among patients with similar international normalized ratio values on warfarin, East Asian patients had the highest risk of intracerebral hemorrhage among other races. ${ }^{33}$ The increase in bleeding risk could be due to smaller average body configurations of East Asians. In a study by Teng et al, ${ }^{18}$ where the PK and PD parameters of ticagrelor were compared in Japanese and Caucasians, the average weight of the volunteers in the two groups were $64 \mathrm{~kg}$ and $77 \mathrm{~kg}$, respectively. When the data were adjusted for body weight, the difference in systemic exposure between the two groups decreased from $40 \%$ to $14 \%$.

Based on the analysis in this review, it was observed that PK parameters of ticagrelor and AR-C124910XX increased dose-proportionally in a predictable manner. However, with the exception of one study, ${ }^{23}$ most PK studies only included around ten subjects per group and this resulted in widespread of the CIs. For example, in Li et al's study, ${ }^{24}$ while the mean plasma concentrations of ticagrelor $60 \mathrm{mg}$ were numerically higher than $45 \mathrm{mg}$, both of their CIs overlapped at all time points, and their differences were not considered to be significant.

Regarding PD parameters, while both low and standard ticagrelor doses alike exhibited much higher antiplatelet activity than clopidogrel in all of the four studies that also included the clopidogrel arm, three studies observed differences between the low- and standard-dose ticagrelor, while two studies observed no differences.

Previous studies ${ }^{4,28}$ have also evaluated the PK/PD characteristics of low dose of ticagrelor compared to the standard dose, albeit mainly in the Caucasian population. The PK and PD findings in these studies involving non-Asian patients are consistent with the findings of this review, where the PK increased in a dose-proportional manner, and this difference may but may not always translate into antiplatelet activity differences.

In terms of clinical efficacy, PEGASUS-TIMI $54^{25}$ observed no statistical difference between the rates of primary efficacy endpoint between two ticagrelor doses (60 mg MD and $90 \mathrm{mg} \mathrm{MD}$ ) after 33 months. Through the subgroup analysis, it was observed that this finding was consistent also in patients from Asia, although it should be interpreted with caution that this subgroup analysis is probably underpowered to detect significant differences among patients from 
different geographical regions of the world. A clinical study by Li et $\mathrm{al}^{21}$ also included only between 108 and 132 patients per group, and it is obvious that the study did not have enough power due to the limited number of subjects.

Therefore, while dose-proportional increases in the PK parameters were observed for ticagrelor, it appears that at both low and standard doses, potent platelet inhibition occurred, which was significantly more than clopidogrel, but the degree of platelet inhibition was very high at both doses that the two different doses of ticagrelor did not always translate into PD differences. There were two clinical studies examining the differences in the outcomes of low and standard doses of ticagrelor in East Asians, but both were limited by the number of included patients. In addition, two studies included different patient populations, in that PEGASUSTIMI $54^{25}$ included patients $1-3$ years post-MI and Li et $\mathrm{al}^{21}$ included patients with unstable angina pectoris and nonrevascularization. Therefore, there are limited evidence available thus far to make a recommendation on clinical efficacy based on ticagrelor doses in East Asian patients.

For safety outcomes, standard dose of ticagrelor, in general, resulted in higher adverse events, including bleeding rates, than the low dose of ticagrelor. . $^{19-21,23,24}$ There were studies $^{19,23,24}$ that reported higher rates of certain adverse events in lower doses, but the differences were mostly contributory to small number of patients included in the study and the differences in rates of bleeding were by only a few patients; therefore, the differences in rates of adverse events cannot be concluded from the currently available studies.

Limitations of this systematic review are as follows. First, this review included only studies that compared standard and low doses of ticagrelor in Asian populations, resulting in inclusion of only a limited number of articles for analysis. Second, not all of the included studies measured all PK, PD, and clinical endpoints; thus, the included studies lacked consistency in the endpoints measured. Lastly, as there were no large-scale, long-term studies on East Asian population (the longest duration was from one study with a median follow-up of 33 months), ${ }^{1}$ it is difficult to predict the results of long-term use of low-dose ticagrelor in East Asian patients.

\section{Conclusion}

In the East Asian population, there is limited evidence available to date that compare low versus standard doses of ticagrelor to evaluate the clinical efficacy and safety. Larger and longer duration studies are warranted to more closely examine the appropriateness of lower doses of ticagrelor in this population.

\section{Acknowledgment}

This work was supported by the National Research Foundation of Korea (NRF) grant funded by the Korea government (MSIT) (No NRF-2017R1C1B1003141).

\section{Disclosure}

The authors report no conflicts of interest in this work.

\section{References}

1. Bonaca MP, Bhatt DL, Cohen M, et al. Long-term use of ticagrelor in patients with prior myocardial infarction. $N$ Engl J Med. 2015; 372(19):1791-1800.

2. Butler K, Teng R. Pharmacokinetics, pharmacodynamics, safety and tolerability of multiple ascending doses of ticagrelor in healthy volunteers. Br J Clin Pharmacol. 2010;70(1):65-77.

3. Zhou D, Andersson TB, Grimm SW. In vitro evaluation of potential drug-drug interactions with ticagrelor: cytochrome $\mathrm{P} 450$ reaction phenotyping, inhibition, induction, and differential kinetics. Drug Metab Dispos. 2011;39(4):703-710.

4. Husted S, Emanuelsson H, Heptinstall S, et al. Pharmacodynamics, pharmacokinetics, and safety of the oral reversible P2Y12 antagonist AZD6140 with aspirin in patients with atherosclerosis: a double-blind comparison to clopidogrel with aspirin. Eur Heart J. 2006;27(9): $1038-1047$.

5. van Giezen JJ, Nilsson L, Berntsson P, et al. Ticagrelor binds to human P2Y(12) independently from ADP but antagonizes ADP-induced receptor signaling and platelet aggregation. J Thromb Haemost. 2009; 7(9):1556-1565.

6. Wallentin L, Becker RC, Budaj A, et al. Ticagrelor versus clopidogrel in patients with acute coronary syndromes. $N$ Engl J Med. 2009;361(11): 1045-1057.

7. Brilinta ${ }^{\circledR}$ (ticagrelor) [package insert]. Wilmington, DE: AstraZeneca L; 2011.

8. Goto S, Huang CH, Park SJ, et al. Ticagrelor vs. clopidogrel in Japanese, Korean and Taiwanese patients with acute coronary syndrome randomized, double-blind, phase III PHILO study. Circ J. 2015;79(11): $2452-2460$.

9. Serebruany V, Tomek A, Pya Y, et al. Inferiority of ticagrelor in the PHILO trial: play of chance in East Asians or nightmare confirmation of PLATO-USA? Int J Cardiol. 2016;215:372-376.

10. Asano T, Kobayashi Y, Fukushima K, et al. Safety and efficacy of lowdose clopidogrel in Japanese patients undergoing coronary stenting, preliminary 30-day clinical outcome. Circ J. 2008;72(10):1707-1708.

11. Fukushima K, Kobayashi Y, Kitahara H, et al. Antiplatelet effect of 50-mg maintenance dose of clopidogrel compared to $200 \mathrm{mg}$ ticlopidine: a preliminary study. Heart Vessels. 2010;25(1):41-44.

12. Ohkubo K, Kobayashi Y. Long-term safety and efficacy of low-dose clopidogrel in Japanese patients undergoing coronary stenting: a preliminary study. Cardiovasc Interv Ther. 2011;26(2):104-108.

13. Yokoi H, Kimura T, Isshiki T, et al. Pharmacodynamic assessment of a novel P2Y12 receptor antagonist in Japanese patients with coronary artery disease undergoing elective percutaneous coronary intervention. Thromb Res. 2012;129(5):623-628.

14. Kimura T, Isshiki T, Ogawa $\mathrm{H}$, et al. Randomized, double-blind, dosefinding, phase II study of prasugrel in Japanese patients undergoing elective percutaneous coronary intervention. J Atheroscler Thromb. 2015; 22(6):557-569.

15. Saito S, Isshiki T, Kimura T, et al. Efficacy and safety of adjusted-dose prasugrel compared with clopidogrel in Japanese patients with acute coronary syndrome: the PRASFIT-ACS study. Circ J. 2014;78(7):1684-1692.

16. [Clopidogrel tablet $25 \mathrm{mg}$ "AA"/Clopidogrel tablet $75 \mathrm{mg}$ "AA"] [PMDA web site]. Available from: https://www.pmda.go.jp/PmdaSearch/iyakuD etail/470007_3399008F1033_2_01. Accessed July 8, 2016. Japanese. 
17. [Effient tablet $2.5 \mathrm{mg} /$ Effient tablet $3.75 \mathrm{mg} /$ Effient tablet $5 \mathrm{mg} /$ Effient tablet $20 \mathrm{mg}$ ] [PMDA web site]. Available from: http://www.info. pmda.go.jp/go/pack/3399009F1020_1_11/. Accessed July 8, 2016. Japanese.

18. Teng R, Butler K. Pharmacokinetics, pharmacodynamics, and tolerability of single and multiple doses of ticagrelor in Japanese and Caucasian volunteers. Int J Clin Pharmacol Ther. 2014;52(6):478-491.

19. Li H, Butler K, Yang L, et al. Pharmacokinetics and tolerability of single and multiple doses of ticagrelor in healthy Chinese subjects: an open-label, sequential, two-cohort, single-centre study. Clin Drug Investig. 2012;32(2):87-97.

20. Li P, Gu Y, Yang Y, et al. Low-dose ticagrelor yields an antiplatelet efficacy similar to that of standard-dose ticagrelor in healthy subjects: an open-label randomized controlled trial. Sci Rep. 2016;24:31838.

21. Li K, Xu Y, Han Q. [Safety and efficacy of low-dose ticagrelor in patients with unstable angina pectoris and non-revascularization]. Chin J New Drugs. 2015;24(21):2480-2484. Chinese.

22. Xue HJ, Shi J, Liu B, et al. Comparison of half- and standard-dose ticagrelor in Chinese patients with NSTE-ACS. Platelets. 2016;27(5): $440-445$.

23. Hiasa Y, Teng R, Emanuelsson H. Pharmacodynamics, pharmacokinetics and safety of ticagrelor in Asian patients with stable coronary artery disease. Cardiovasc Interv Ther. 2014;29(4):324-333.

24. Li H, Guo J, Carlson GF, et al. Pharmacodynamics, pharmacokinetics, and safety of ticagrelor in Chinese patients with stable coronary artery disease. Br J Clin Pharmacol. 2016;82(2):352-361.

25. Bonaca MP, Bhatt DL, Storey RF, et al. Ticagrelor for prevention of ischemic events after myocardial infarction in patients with peripheral artery disease. J Am Coll Cardiol. 2016;67(23):2719-2728.
26. Ahmad T, Voora D, Becker R. The pharmacogenetics of antiplatelet agents: towards personalized therapy? Nat Rev Cardiol. 2011;8(10): 560-571.

27. Wang H, Wang X. Efficacy and safety outcomes of ticagrelor compared with clopidogrel in elderly Chinese patients with acute coronary syndrome. Ther Clin Risk Manag. 2016;12:1101-1105.

28. Storey RF, Angiolillo DJ, Bonaca MP, et al. Platelet inhibition with ticagrelor $60 \mathrm{mg}$ versus $90 \mathrm{mg}$ twice daily in the PEGASUS-TIMI 54 Trial. J Am Coll Cardiol. 2016;67(10):1145-1154.

29. Jeong YH. "East asian paradox": challenge for the current antiplatelet strategy of "one-guideline-fits-all races" in acute coronary syndrome. Curr Cardiol Rep. 2014;16(5):485.

30. Levine GN, Jeong YH, Goto S, et al. Expert consensus document: World Heart Federation expert consensus statement on antiplatelet therapy in East Asian patients with ACS or undergoing PCI. Nat Rev Cardiol. 2014;11(10):597-606.

31. Lutsey PL, Cushman M, Steffen LM, et al. Plasma hemostatic factors and endothelial markers in four racial/ethnic groups: the MESA study. J Thromb Haemost. 2006;4(12):2629-2635.

32. Kelley-Hedgepeth A, Lloyd-Jones DM, Colvin A, et al. Ethnic differences in C-reactive protein concentrations. Clin Chem. 2008;54(6): 1027-1037.

33. Shen AY, Yao JF, Brar SS, et al. Racial/ethnic differences in the risk of intracranial hemorrhage among patients with atrial fibrillation. $J \mathrm{Am}$ Coll Cardiol. 2007;50(4):309-315. 


\section{Supplementary material}

Search terms used for the literature search.

("ACS" OR "acute coronary syndrome" OR "myocardial infarction" OR "MI" OR "NSTEMI" OR "non-ST elevated myocardial infarction" OR "non ST elevated myocardial infarction" OR "STEMI" OR "ST-elevated myocardial infarction" OR "ST elevated myocardial infarction" OR "NSTEACS" OR "unstable angina" OR "UA" OR "percutaneous coronary intervention" OR "PCI" OR "CAD” OR “coronary artery disease" OR "coronary arteriosclerosis" OR “coronary atherosclerosis" OR "angina pectoris" OR "stable angina" OR "healthy" OR volunteer OR participant OR subject OR Asian OR "Japanese" OR "Chinese" OR "Korean") AND ("low dose" OR "low-dose" OR "half dose" OR "half-dose" OR "one-quarter standard-dose" OR "standard dose" OR " $22.5 \mathrm{mg}$ " OR “45 mg” OR “50 mg” OR “60 mg” OR “multiple doses") AND (ticagrelor OR Brilinta OR Brilique OR "P2 $\mathrm{Y}_{12}$ receptor antagonist" OR "P2 $\mathrm{Y}_{12}$ inhibitor" OR “AR-C124910XX”).

\section{Publish your work in this journal}

Therapeutics and Clinical Risk Management is an international, peerreviewed journal of clinical therapeutics and risk management, focusing on concise rapid reporting of clinical studies in all therapeutic areas, outcomes, safety, and programs for the effective, safe, and sustained use of medicines. This journal is indexed on PubMed Central, CAS,
EMBase, Scopus and the Elsevier Bibliographic databases. The manuscript management system is completely online and includes a very quick and fair peer-review system, which is all easy to use. Visit http://www.dovepress.com/testimonials.php to read real quotes from published authors.

Submit your manuscript here: http://www.dovepress.com/therapeutics-and-clinical-risk-management-journal 